\section{DURABILITY AND EASY HANDLING AS STANDARD}

For perfect, natural looking restorations, choose Ceram.X duo+ from DENTSPLY. Ceram.X duo+ is everything a modern dentist should expect from

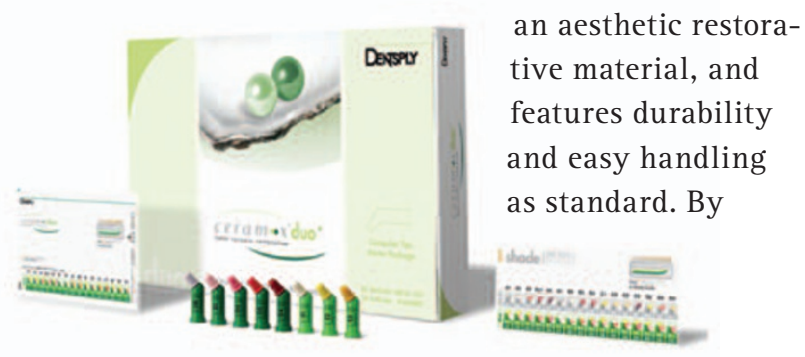

combining both organic and inorganic structures, Ceram.X duo+ allows the clinician to create natural aesthetics the simple way, using a twolayer system with a minimum number of shades to meet all patient profiles.

Among its chief benefits, Ceram.X duo+ exhibits high fracture toughness for longer- lasting restorations and also features an innovative inhibitor system for longer working time of up to 180 seconds before light curing.

Visit www.dentsply.co.uk or call 0800072 3313. Earn rewards against purchases at dentsplyrewards.co.uk. Access webinars and products demonstrations and earn CPD at dentsplyacademy.co.uk.

\section{LOOK NO FURTHER FOR RESTORATIVE MATERIALS}

Plandent stock a huge range of dental restorative materials. Whether you are looking for one of the latest, technologically advanced composite materials on the market or the amalgam that you have been using for some time, look no further.

Plandent keep in stock a full range of the leading brands of restorative dental materials: glass ionomers, compomers, composites, temporary cements or any of the accessories that you use on a daily basis. Many of these restorative materials are regularly promoted at extra special prices - call now for details.

Due to the group buying power of Plandent and efficient supply chain, Plandent offer price reductions against recommended dental prices, meaning a great deal for you.

Whether you're looking for dental consumables, a new handpiece or capital equipment, Plandent offer you everything you could possibly need for your surgery. All equipment sales are fully backed by Plandent's comprehensive after sales customer care programme.

For further information Freecall 0500500322.

\title{
A BREAKTHROUGH TREATMENT FOR DENTINE HYPERSENSITIVITY
}

On 18 July GlaxoSmithKline (GSK) Consumer

Healthcare held a NovaMin Symposium at the Royal Institution of Great Britain in London.

Speakers David C. Greenspan, the co-inventor of NovaMin, and Dr Teresa Layer, the Vice-President of Oral Health R\&D at GSK, presented on GSK's breakthrough technology for treatment of dentine hypersensitivity: calcium sodium phosphosilicate - or NovaMin - and on GSK's oral health mission 'to make the world's mouths healthier'. Stephen Hancocks OBE was chair for the occasion.

NovaMin is unique in that it is the first material that will repair the vulnerable areas of sensitive teeth using the natural building blocks of teeth, calcium and phosphorus. It is a breakthrough science that offers a real benefit for patients who suffer with hypersensitivity.

NovaMin bonds to exposed dentine, which is where the problem is in hypersensitive patients,

\section{EASY SCANS OF THE ORAL CAVITY}

CEREC Omnicam generates full colour scans of intraoral surfaces without the need for a powder coating. Operating the camera is simple and intuitive. The imaging functionality and camera dimensions have been ergonomically designed.

The CEREC Omnicam supports video streaming; it digitises the structures of the jaw in their natural colour; and it does not require a powder coating of the tooth surfaces.

The CEREC Omnicam is extremely resistant to camera shake and it's not necessary to apply a powder coating to the teeth. Instead the user moves the camera head over the intraoral surfaces in a smooth, flowing movement. A virtual 3D model is displayed in natural colour on the monitor of the CEREC AC. Thanks to this lifelike visualisation, it is easy to distinguish between different materials and identify the palatinal and gingival contours. As a result it is easy to navigate around the oral cavity and deter- and forms a reparative layer which occludes the tubules and decreases the movement of intratubular fluid and the signals of pain. It also forms a reparative layer made from hydroxyapatite, which is harder than the underlying dentine.

NovaMin powers GSK's brand new toothpaste, Sensodyne Complete Protection.

The NovaMin Symposium was a highly successful evening with excellent feedback from the delegates who attended.

www.sensodyne.co.uk/products/sensodyne-products/sensodyne-complete-protection.aspx mine the preparation margin.

The extensive depth of field of CEREC Omnicam delivers high-precision images, even in cases where the camera has been placed directly on the tooth. Thanks to the slimline design and the compact camera head, it is easy to scan inaccessible areas such as the distal surfaces of the posterior molars.

In addition, the CEREC Omnicam boasts a patient counselling mode. The dentist can record short video clips and present these to the patient via the CEREC AC monitor.

Call Sirona Dental Systems on 08450715040 or email info@ sironadental.co.uk.

www.sironadental.co.uk 